\title{
“Los lados de un polígono no se intersecan”... Ahondar en la voz de los estudiantes
}

\author{
"The sides of a polygon do not intersect" ... Delving into the voice of the \\ students
}

Leonor Camargo*

ORCID iD 0000-0002-2237-7306

Patricia Perry $^{* *}$

ORCID iD 0000-0002-9162-071X

\begin{abstract}
Resumen
Caracterizamos expresiones discursivas de tres estudiantes de grado sexto sobre la frase se intersecan y el efecto que tienen en la construcción de significados colectivos y compartidos de intersección. Empleamos una estrategia cualitativa basada en prácticas usuales, para registrar información, fragmentarla sin perder el contexto donde se enmarca la interacción, y seleccionar expresiones para su análisis. Siguiendo técnicas propias de la etnometodología, el análisis se hizo en términos de: (i) inteligibilidad y autenticidad, (ii) rasgos del discurso propuestos por Sfard y (iii) nuestra conceptualización de voz colectiva y voz compartida. Encontramos un aula de geometría en la que las voces de estudiantes se construyen de forma colectiva y generan significado compartido sobre la intersección que se refleja en una transición discursiva puntual. El aporte de este documento reside en el uso de los rasgos discursivos considerados en el análisis para enfocar la mirada al discurso en el contenido matemático.
\end{abstract}

Palabras clave: Voz de los Estudiantes. Aprender visto como Desarrollar un Discurso. Rasgos del Discurso Matemático. Operación de Intersección.

\begin{abstract}
We characterized both discursive expressions of three sixth grade students on the phrase intersect each other and the effect they have on the construction of collective and shared meanings of intersection. Recording data, extracting information without losing the context where the interaction is framed, and selecting expressions to analyze were all done by using a classroom-based qualitative strategy. The analysis, following techniques typical of ethnomethodology, was done in terms of: (i) intelligibility and authenticity, (ii) features of the discourse proposed by Sfard and (iii) our conceptualization of collective voice and shared voice. We found a geometry classroom in which student voices are collectively constructed and generate shared meaning about intersection, which is reflected in a punctual discursive transition. The contribution of this paper resides in the use of the discursive features regarded in the analysis to focus the gaze to the discourse on mathematical content.
\end{abstract}

\footnotetext{
* Doctora en Didáctica de las Matemáticas, Universidad de Valencia (UV). Profesora de Planta, Universidad Pedagógica Nacional (UPN), Bogotá, Cundinamarca, Colombia. Dirección postal: Calle 64, No. 4-22, Apartamento 602, Bogotá, Colombia, C.P: 110231. E-mail: lcamargo@ pedagogica.edu.co.

** Licenciada en Educación con especialidad en Matemáticas, Universidad Pedagógica Nacional (UPN). Investigadora, Universidad Pedagógica Nacional (UPN - CIUP), Bogotá, Cundinamarca, Colombia. Dirección postal: Carrera 45A, No. 123-64, Apartamento 301, Bogotá, Colombia, C.P: 111111. E-mail: pperryc@yahoo.com.mx.
} 
Keywords: Students voice. Learning as Developing a Discourse. Features of Mathematical Discourse. Intersection Operation.

\section{Introducción}

En nuestro ejercicio investigativo partimos del supuesto, hoy ampliamente aceptado en el campo de la Educación, de que el lenguaje y la comunicación desempeñan un papel preponderante en la construcción de significado. Específicamente, el lenguaje matemático más que una forma de representación del pensamiento es el principal recurso cultural a través del cual se aprende, pues las matemáticas mismas son una forma de comunicación con otros o con uno mismo (SFARD, 2008).

Diversas investigaciones se han enfocado en el análisis de interacciones discursivas de los estudiantes en la clase de matemáticas. Los trabajos se han dirigido principalmente hacia la participación discursiva, sin referirse al contenido específico. Por ejemplo: Walshaw y Anthony (2008), Wood y Kalinec (2012), Esmonde y Langer-Osuna (2013) y Jung y Shütte (2018) se centran en la búsqueda de estrategias de enseñanza para favorecer la participación discursiva de los estudiantes en condiciones de equidad; Barwell (2013) y Clarke, Xu y Wan (2013) se interesan por las ocasiones que tienen los estudiantes de escuchar y decir términos matemáticos; Jablonka (2006), Cao et al. (2013) y Kaur (2013) e Ingram, Andrews y Pitt (2019) examinan la expresión pública de los estudiantes, buscando que ellos compartan sus pensamientos con sus compañeros con apoyo del profesor o autónomamente.

El problema al que atiende la investigación ${ }^{1}$, de la cual este artículo es producto, se esboza a continuación. De acuerdo con investigaciones como las referenciadas en el párrafo anterior, en las últimas décadas ha sido una práctica común en el aula de matemáticas que los estudiantes hablen entre ellos o con el profesor sobre asuntos matemáticos que se tratan en clase, pero no hay suficientes evidencias que permitan afirmar que la interacción que se promueve brinde oportunidades para que ellos produzcan discursos que les permitan contrastar sus planteamientos con los de sus compañeros, construir significado y refinar sus ideas sobre tales asuntos. Lo anterior porque pocos estudios en la línea del lenguaje entran en detalles sobre la construcción de significado de objetos, relaciones u operaciones matemáticas específicas.

En nuestra investigación nos enfocamos en clases de geometría y nos proponemos caracterizar, en diversas clases de nivel escolar, expresiones discursivas de estudiantes

\footnotetext{
${ }^{1}$ Los resultados que se comunican provienen de la investigación adelantada con financiación del Centro de Investigaciones de la Universidad Pedagógica Nacional (CIUP) en los proyectos Voces de los estudiantes en la clase de geometría (DMA-461-18) y Gestión de voces de los estudiantes en la clase de geometría (DMA-489-19).
} 
indagando si es posible considerarlas auténticas e inteligibles. Si es el caso, pretendemos ver si tienen o no alguna influencia en la construcción colectiva de significados compartidos sobre objetos, operaciones o relaciones matemáticas. En este artículo nos enfocamos en expresiones relacionadas con la frase se intersecan. Pretendemos argumentar que las expresiones analizadas contribuyen a la construcción colectiva de significado de la operación matemática de intersección. Los resultados de la caracterización hecha nos inducen a promover la difusión de dinámicas discursivas en pro del aprendizaje.

\section{Marco conceptual}

Estructuramos el marco conceptual en cuatro secciones en las que informamos sobre los aspectos tenidos en cuenta para conformar las categorías de análisis. En el apartado sobre el método especificamos algunos descriptores para cada una de ellas que orientan el análisis.

\subsection{Aprendizaje de las matemáticas}

La perspectiva teórica sociocultural en la que se inscribe la investigación ha cobrado relevancia desde los años 90 del siglo XX. Ha generado posturas sobre el aprendizaje que lo ven como el proceso de construcción de significado en prácticas discursivas propias de las matemáticas (BEN-ZVI; SFARD, 2007; KAUR, 2013; RADFORD; BARWELL, 2016; JUNG; SHÜTTE, 2018). Para autores como Walshaw y Anthony (2008) y Kaur (2013) los escenarios de aprendizaje se caracterizan por promover el discurso como elemento instruccional relevante. Por ello, hoy, el desarrollo de la comunicación es uno de los objetivos de la educación matemática.

Bajo ese punto de vista, entendemos que aprender matemáticas es un proceso de transición discursiva que va desde un estado en el que predominan expresiones del uso común de la lengua natural para comunicar ideas hacia la participación, de manera cada vez más inclusiva, en un discurso especializado para comunicarse en formas reconocibles histórica y culturalmente como matemáticas (SFARD, 2008; RADFORD; BARWELL, 2016). Reconocemos que, en un momento dado y quizá por un tiempo considerable, los estudiantes pueden no hacer uso especializado de la lengua natural para comunicarse de manera eficaz sobre matemáticas; sin embargo, su lenguaje es un punto de partida para la construcción de significado de objetos matemáticos y ejerce una clara influencia en la conceptualización y en el desempeño asociado. 
Vemos las clases de matemáticas como espacios de participación discursiva donde los estudiantes deberían tener la oportunidad de realizar, junto con compañeros y profesor, actividades matemáticas, expresar significados personales (de objetos, situaciones, tareas, ideas, conceptos, procesos etc.), responder reflexivamente a los significados comunicados por otros, y construir discursos matemáticos, haciendo uso común o especializado de su lengua. Entendemos que el significado personal de un objeto es una red de ideas, creencias, imágenes del objeto, de las relaciones de este con otros objetos y de las formas válidas para representar, discernir, pensar, usar y hablar sobre este. El significado se construye individual y colectivamente en el intercambio comunicativo.

\subsection{Voz matemática del estudiante}

De las diversas verbalizaciones del estudiante en el aula de matemáticas, denominamos voz matemática del estudiante (en adelante, voz del estudiante) a la expresión oral, escrita o gestual, auténtica e inteligible, que este emite en interacción con el profesor u otros estudiantes, para comunicar una idea (en calidad de afirmación, inquietud, pregunta, explicación etc.) sobre un asunto matemático acerca del cual versa la clase. El adjetivo auténtica pretende destacar el carácter no impostado de la expresión, que permite reconocerla como propia de quien la emite y en la que se identifica un compromiso personal.

El adjetivo inteligible quiere aclarar que la expresión que es voz del estudiante es interpretable por quien la recibe. La intención con la que se emite una voz no siempre es explícita, pero sí puede ser rastreable mediante pistas comunicativas, en el contexto de una interacción; debería generar un cierto tipo de reacción comunicativa que está en sintonía con la intención con la que se emite.

Siguiendo a Morgan y Sfard (2016), los siguientes son rasgos característicos interrelacionados de cualquier expresión matemática discursiva:

Vocabulario y sintaxis. Palabras empleadas y uso de estas. Se caracterizan por: (i) la especialización del lenguaje, que depende de la inclusión de palabras, frases u oraciones propias de las matemáticas, de la construcción gramatical empleada y del uso dado al lenguaje especializado; (ii) la complejidad lógica discursiva, en términos del tipo y la frecuencia de uso de cuantificadores o conectivos lógicos.

Mediador visual. Objeto visible usado por quien se expresa para identificar a qué objeto matemático (inexistente) se refiere; hace parte integral de su comunicación. Se caracteriza en términos de: la existencia independiente del discurso que median, las circunstancias de uso y 
de dónde proviene.

Rutina. Manera repetitiva distintiva de llevar a cabo una actividad matemática, que obedece a un conjunto de reglas y que limita lo que es posible hacer (por ejemplo, definir un objeto geométrico o demostrar un teorema). El uso de vocabulario y mediadores visuales, así como el proceso de creación y explicitación de una narrativa pueden hacerse de maneras rutinarias; en ese sentido, Morgan y Sfard (2016) señalan que la rutina, como rasgo distintivo, eventualmente se solapa con los demás. Una vez identificada una rutina, puede caracterizarse en función del uso que hace de esta quien se comunica, del grado de complejidad, de las circunstancias en las que se usa y el momento en que deja de usarse.

Narrativa. Relato o secuencia de verbalizaciones, acerca de objetos, de relaciones entre objetos o de procesos matemáticos. Su caracterización se puede hacer en función de los recursos expresivos mediante los cuales se hace la enunciación. Por ejemplo, (i) en el uso de sustantivos matemáticos para encapsular acciones y procesos en objetos (en lugar, por ejemplo, del uso de deícticos, adjetivos o adverbios); (ii) en el protagonismo dado a los objetos matemáticos por encima de las acciones personales; (iii) en la explicitación o denominación de objetos matemáticos presentes en acto durante la comunicación; (iv) en los modificadores gramaticales que indican el grado de certidumbre que se tiene sobre lo dicho.

\subsection{Voz colectiva y voz compartida en la clase de matemáticas}

En la clase, cada miembro de la comunidad del aula, incluso el profesor, construye significado a través de un proceso interpretativo en el que los significados comunicados por otros ejercen una influencia importante. En busca de identificar el posible aporte de la voz de los estudiantes a la construcción de significado, recurrimos a dos adjetivos para calificar la voz: colectiva y compartida.

Usamos voz colectiva para referirnos a una voz en cuya formulación han participado de manera sustancial en términos de proponer o de elaborar, dos o más personas presentes en el discurso en el que surge la voz. Aunque un estudiante asuma el papel protagónico en la formulación, puede entreverse que otros miembros de la clase contribuyen a darle forma.

Usamos voz compartida para referirnos a una voz que representa a otras voces surgidas en la clase, por cuanto todas ellas guardan similitud sintáctica o semántica. Es posible que esta similitud se dé sin que sea evidente una voz colectiva. Esto puede suceder cuando los estudiantes han tenido alguna experiencia previa común, social o académica, que los lleva a proferir elaboraciones similares. Pero, también puede suceder que la similitud sea fruto de 
la voz construida colectivamente.

\subsection{Construcción de significado de intersección}

En términos de los rasgos del discurso sugeridos por Sfard (2008), puntualizamos aspectos de la intersección y de la expresión se intersecan - objetos del discurso matemático.

\subsubsection{Relativos al vocabulario y la sintaxis}

El término intersección, en geometría, es la forma especializada para referir a dos asuntos. Uno, a una operación entre dos objetos geométricos específicos que tiene como resultado el conjunto de puntos que pertenecen a ambos objetos. Al usar esta acepción se dice, por ejemplo, la intersección de la recta $l$ y la circunferencia $C$ es el conjunto formado por los puntos $M$ y $N$. Dos, a una relación entre dos objetos geométricos que los vincula describiéndolos en términos de si tienen puntos en común. Al usar esta acepción se dice, por ejemplo, la recta $m$ tiene intersección no vacía con la recta $n$. La relación tener intersección no vacía se puede expresar también como: la recta $m$ es intersecante con la recta $n$ o las rectas $m$ y $n$ son intersecantes. Sobre esta relación se puede predicar como se hace con otras relaciones geométricas como el paralelismo o la semejanza. Por ejemplo, se puede decir que la relación tener intersección no vacía entre rectas es reflexiva y simétrica, pero no es transitiva.

En los primeros cursos de geometría plana en la escuela, el término se introduce generalmente ligado a la primera acepción. Las expresiones usuales acerca de la operación de intersección entre rectas son: Al hacer la intersección entre la recta $l$ y la recta $m$ se determina el punto $P$; en este caso, la atención se enfoca en el resultado de la operación, ejecutada por un sujeto. La recta $l$ interseca a la recta $m$; en este caso, hay implicada una acción-proceso de una recta sobre otra. La recta $l$ y la recta $m$ se intersecan (i.e., el conjunto resultante no es vacío); en este caso, hay implicada una acción recíproca de las rectas.

\subsubsection{Relativos a los mediadores visuales}

La comunicación sobre la operación de intersección suele acompañarse de representaciones geométricas donde sobresalen los puntos de intersección (bien sea porque se resaltan o porque se nombran) y de notación donde aparece el símbolo $\cap$. Infortunadamente, en 
los primeros años de secundaria, al trabajar la intersección principalmente entre rectas, las representaciones geométricas frecuentes suelen restringirse a dos rectas que forman una cruz y no se introducen otras representaciones que podrían contribuir a la construcción de significado.

\subsubsection{Relativos a las rutinas y las narrativas}

En geometría, los siguientes procedimientos rutinarios están ligados a la operación de intersección: reproducir la representación de un ejemplo prototípico (rutina que no da suficiente información para identificar qué significado se tiene de la expresión que se intersequen); trazar una línea, determinar un punto de ella y trazar otra línea que contenga al punto (rutina que podría estar asociada a la expresión la segunda línea interseca a la primera); hacer un escaneo perceptual de la representación de dos líneas en busca de uno o más puntos que pertenezcan a ambas líneas (rutina que podría estar ligada a la expresión estas líneas se intersecan); determinar un punto que será la intersección de dos líneas y luego trazar dos líneas que pasen por dicho punto (rutina posiblemente asociada a una expresión como el punto $P$ es común a estas lineas).

Narrativas sobre la operación de intersección pueden referirse a condiciones propias de los procedimientos que acabamos de formular. Por ejemplo: la intersección siempre se realiza entre dos objetos; para más de dos objetos, la operación se hace reiteradamente; el punto en común da lugar a un criterio para evaluar si dos líneas se intersecan o aporta una condición para construir rectas intersecantes. Una narrativa relacionada con la operación de intersección, que sería deseable que surgiera en la transición discursiva debería formular que la intersección es una operación realizable entre dos objetos geométricos cualesquiera.

\section{Método}

De acuerdo con el propósito de la investigación, adoptamos una estrategia investigativa basada en prácticas usuales (CAMARGO, en evaluación) y usamos técnicas propias de la etnometodología para el análisis de expresiones discursivas (INGRAM, 2018). Observamos y registramos el desarrollo de algún contenido del currículo en cinco aulas de matemáticas, de los grados $5^{\circ}$ a $9^{\circ}$ de Educación Básica. En particular, en el análisis que presentamos aquí, la información proviene de dos sesiones de clase de grado $6^{\circ}$ en un colegio de Bogotá.

Con la autorización debida para el caso, registramos la interacción comunicativa. Para ello, dos investigadoras del equipo accionaron tres cámaras de video (una fija y dos móviles) y 
tres grabadoras de audio. Intentamos captar la mayor información sobre los sucesos de la clase. Las grabaciones fueron transcritas en su totalidad y alimentamos los textos con fotografías y aclaraciones recogidas en notas de campo.

Para obtener los datos investigativos seguimos una ruta similar a la sugerida por Villalta (2009) para analizar conversaciones en clase. Este autor sugiere cómo fragmentar, reducir y depurar las transcripciones hasta obtener un conjunto de intervenciones de interés investigativo, enmarcadas en intercambios comunicativos, momentos de la clase y el cuadro comunicativo en general. Por esta vía, identificamos las expresiones que analizamos como potenciales voces de los estudiantes. Todas ellas se caracterizan por ser actos comunicativos, centrados en las matemáticas que se producen en la clase de geometría.

La herramienta analítica fue construida por el equipo de investigación en un ejercicio académico de contraste bidireccional, entre la teoría y los datos que íbamos obteniendo en función de los propósitos del estudio. Este proceso nos llevó a establecer tres conjuntos de categorías de análisis. El primero, para decidir si las expresiones candidatas a voz efectivamente lo son (Cuadro 1).

\begin{tabular}{|c|l|}
\hline Categoría & \multicolumn{1}{|c|}{ Ejemplo de descriptores } \\
\hline Inteligibilidad & $\begin{array}{l}\text { Se identifican ideas matemáticas sobre el tema de la conversación: (i) Hay una } \\
\text { construcción gramaticalmente aceptable en castellano. (ii) Es posible interpretar de } \\
\text { manera sustentada lo que expresa el estudiante. }\end{array}$ \\
\hline Autenticidad & $\begin{array}{l}\text { Se encuentran indicios de elaboración propia: (i) Presencia de términos idiosincráticos. } \\
\text { (ii) Exposición de ideas propias o puntos de vista. (iii) Paráfrasis en términos propios. }\end{array}$ \\
\hline
\end{tabular}

$$
\begin{gathered}
\text { Cuadro } 1 \text { - Rasgos para voz - Categorías de análisis } \\
\text { Fuente: elaborado por el autor }
\end{gathered}
$$

Si una expresión es voz, usamos el segundo conjunto de categorías (Cuadro 2) para determinar sus rasgos, adaptando y complementando la propuesta de Morgan y Sfard (2016).

\begin{tabular}{|c|l|}
\hline \multicolumn{2}{|c|}{ Vocabulario } \\
\hline $\begin{array}{c}\text { Especialización del } \\
\text { vocabulario }\end{array}$ & $\begin{array}{l}\text { (i) Uso de palabras, frases, oraciones especializadas. (ii) Construcción gramatical } \\
\text { con la sintaxis aceptada. }\end{array}$ \\
\hline $\begin{array}{c}\text { Objetificación del } \\
\text { discurso }\end{array}$ & $\begin{array}{l}\text { (i) Inclusión del ser humano como agente de las acciones que se comunican. (ii) } \\
\text { Uso de sustantivos matemáticos especializados para encapsular operaciones o } \\
\text { procesos en relaciones u objetos. (iii) Explicitación de objetos matemáticos } \\
\text { presentes en acto. }\end{array}$ \\
\hline $\begin{array}{c}\text { Complejidad lógica } \\
\text { (i) Tipo y frecuencia de conjunciones, disyunciones, implicaciones y negaciones. } \\
\text { (ii) Uso de cuantificadores. }\end{array}$ \\
\hline $\begin{array}{c}\text { Presencia de mediadores } \\
\text { visuales }\end{array}$ & $\begin{array}{l}\text { Uso de objetos perceptuales en el discurso y circunstancias del uso: (i) } \\
\text { Representaciones geométricas. (ii) Iconos gestuales o escritos. (iii) Notación. }\end{array}$ \\
\hline \multicolumn{2}{|c|}{ Rutinas } \\
\hline Acciones rutinarias & Presencia de acciones matemáticas que siguen patrones. \\
\hline
\end{tabular}




\begin{tabular}{|c|l|}
\hline $\begin{array}{c}\text { Características de las } \\
\text { acciones rutinarias }\end{array}$ & $\begin{array}{l}\text { Rasgos característicos de las rutinas empleadas: (i) Complejidad. (ii) } \\
\text { Circunstancias en las que se usa. (iii) Utilidad de la rutina. }\end{array}$ \\
\hline \multicolumn{2}{|c|}{ Narrativas } \\
\hline Sobre qué es la narrativa & (i) Operaciones o procesos, (ii) procedimientos, (iii) objetos. \\
\hline $\begin{array}{c}\text { Tipo de narrativa según } \\
\text { grado de certidumbre }\end{array}$ & $\begin{array}{l}\text { Afirmaciones categóricas, preguntas, enunciados dubitativos, enunciados } \\
\text { condicionales. }\end{array}$ \\
\hline
\end{tabular}

Cuadro 2 - Rasgos de la voz - Categorías de análisis

Fuente: elaborado por el autor

Además de caracterizar la voz, para determinar su papel en la construcción compartida y colectiva de significado empleamos un tercer conjunto de categorías (Cuadro 3).

\begin{tabular}{|c|l|}
\hline $\begin{array}{c}\text { Inteligible para } \\
\text { participantes en el } \\
\text { discurso }\end{array}$ & $\begin{array}{l}\text { Reacciones visibles que son indicios de que la voz es interpretada por otros } \\
\text { miembros de la clase (manifestaciones de aprobación, entendimiento, } \\
\text { objeciones etc.). }\end{array}$ \\
\hline Compartida & $\begin{array}{l}\text { Representatividad de la voz por su similitud sintáctica o semántica con otras } \\
\text { voces. }\end{array}$ \\
\hline Colectiva & $\begin{array}{l}\text { Contribución, de manera sustancial, de más de un participante en la formulación } \\
\text { de la voz (aunque un participante asuma el papel protagónico en la } \\
\text { formulación). }\end{array}$ \\
\hline
\end{tabular}

Cuadro 3 - Rasgos para el efecto de la voz - Categorías de análisis

Fuente: elaborado por el autor

Los descriptores incluidos en cada cuadro son pistas útiles para analizar las expresiones en el contexto comunicativo, pero no pretenden ser indicadores exhaustivos ni constituirse en rótulos específicos de cada expresión. El análisis adopta técnicas etnometodológicas (INGRAM, 2018) que reconocen la necesidad de ofrecer posibles interpretaciones situadas, desde la perspectiva de quien las emite e influidas por otras expresiones discursivas que las acompañan. Las interpretaciones de los investigadores son las que suponen tienen los participantes del discurso, aunque no sean conscientes de ello o no estén totalmente explícitas, pero sí se revelan en la interacción. Lo anterior hace que el análisis de las expresiones no se reduzca a la asignación de códigos correspondientes a las categorías, sino que obliga a los investigadores a proponer descripciones particulares de las interpretaciones que suponen.

\section{Análisis}

\subsection{Contexto comunicativo}

El curso de geometría tiene una intensidad horaria de dos horas semanales y se desarrolla en un aula dotada de treinta tabletas digitales, que se vinculan a un tablero inteligente. Los 28 estudiantes se distribuyen en mesas de cuatro puestos.

En general, el ambiente comunicativo es de confianza y el profesor gestiona la clase 
impulsando la participación discursiva. No descalifica ninguna intervención, no coarta la expresión de ideas, aunque no sean las esperadas o las "correctas", exige de los estudiantes el respeto por las ideas de los demás $\mathrm{y}$, casi siempre, da igual valor a las ideas de los estudiantes que a las que él propone. Los estudiantes son bastante libres de intervenir o no, aunque el profesor los invita con frecuencia a que lo hagan.

\subsection{Constitución del tema de conversación}

La observación coincide con el inicio del segundo periodo escolar. El profesor nos dice, en reunión previa a la grabación de las clases, que se centrará en los temas de polígonos y triángulos, y que en el periodo inmediatamente anterior estudiaron relaciones entre rectas y clases de ángulos. El tema de las dos clases observadas y registradas, declarado por el profesor, es "Polígonos". Él esperaba que los estudiantes tuvieran un acercamiento a la definición de polígono y la usaran en la resolución de un problema relacionado con una trayectoria.

En el transcurso de las dos clases, hubo varios temas de conversación: ¿los polígonos tienen "relleno"?², ¿sus lados se intersecan?, ¿sus lados “cambian de dirección”? En el presente análisis nos enfocamos en algunos intercambios sobre la intersección de los lados de un polígono, por ser un tema recurrente. En ese sentido, aunque el tema de la clase era polígonos, el tema de conversación en el que se enmarcan las expresiones que analizamos es intersección de los lados de un polígono.

Es importante enfocarnos en la intersección pues al rastrear lo que los estudiantes dicen o escriben notamos que el proceso de construcción de significado de esta operación es más gradual de lo que se supone y solo si los escuchamos podremos apoyar útilmente su aprendizaje.

En las dos clases observadas, catorce estudiantes expresan, motu proprio, lo que entienden por se intersecan o intersecarse, y van construyendo un significado de segmentos o lados intersecantes, gracias a la interacción comunicativa en la que el profesor juega un papel clave.

\subsection{Intercambio comunicativo}

En el proceso de construcción de datos investigativos hallamos expresiones de los estudiantes, que aluden a la operación de intersección al hacer referencia a los lados de un

\footnotetext{
${ }^{2}$ Esta alusión fue hecha porque al trabajar con no ejemplos de polígono, el profesor incluyó una región poligonal.
} 
polígono, candidatas a ser consideradas voz. En este texto, nos enfocamos en los discursos de Gabriela, Tomás y Nicolás al abordar la tarea de mencionar rasgos de los polígonos y posteriormente escribir una definición de polígono. Enseguida, relatamos el intercambio comunicativo que contextualiza sus intervenciones.

Tras identificar y anotar rasgos de ejemplos y no ejemplos de polígonos, los estudiantes los comunican oralmente, a solicitud del profesor. Nueve estudiantes piden turno para participar. El profesor da la palabra y anota en el tablero los rasgos mencionados (e.g., "no tener curvas", "no tener líneas en el interior", "representarse con letras").

Refiriéndose tácitamente a los lados, Gabriela dice: "no se intersecan" y haciendo una $\mathrm{X}$ con los dedos índices a la vez que dice: "sus líneas no", explicita, en respuesta al profesor, lo que entiende por no intersecarse. El profesor representa en el tablero dos segmentos $(\overline{S T}$ y $\overline{T R})$ (Figura 1) y pregunta a los estudiantes si se intersecan.

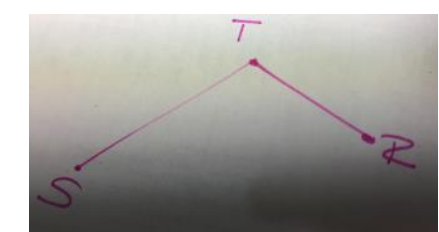

Figura 1 - Representación del profesor para analizar si los segmentos se intersecan Fuente: elaborada por el autor

Para responder la pregunta del profesor, tras su verbalización, "No tiene un punto en común para”, Tomás la completa haciendo una cruz con los dedos índices; luego, para explicitar lo que entiende por intersecarse alude al punto que queda al cruzarse las líneas y lo señala recurriendo a gestos con los dedos y la boca (Figura 2). Valiéndose de gestos de cabeza y manos (Figura 3), Nicolás muestra que va siguiendo la conversación en curso y que está de acuerdo con lo dicho; pero al escuchar la expresión "punto en común”, proferida por Tomás, un nuevo gesto de manos (Figura 4) hecho por Nicolás indica una nueva comprensión.

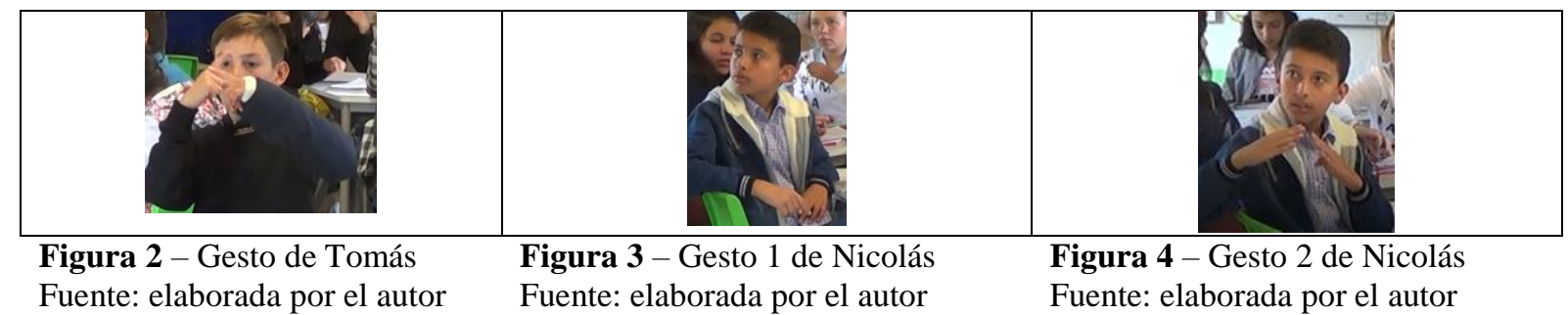

Al intervenir de nuevo, Gabriela, con gestos de brazos y dedos, complementa su verbalización en la que dice que los segmentos "no se cortan así sino así”. Después, refiriéndose a qué entiende por intersecar, pregunta hecha por el profesor, la estudiante dice evocar que "un polígono no tiene líneas afuera. O sea, que no... que no pasaba de ese punto”, y para aclararle a 
Tomás la idea expresada, dice: “pues que no sobresalen más líneas después del punto común”.

Con miras a sacar conclusiones de la interacción discursiva, el profesor vuelve a preguntar si hay o no intersección de los segmentos. Varios estudiantes, en coro, responden afirmativamente; Nicolás precisa que se intersecan en el punto T. El profesor invita a Gabriela a revisar su respuesta inicial; ella afirma que los lados del polígono: "sí se intersecan, pero no van más allá del punto que tienen en común”.

En suma, las expresiones de Gabriela, Tomás y Nicolás se intercalan y se influyen mutuamente. Incluso, hay intervenciones de otros estudiantes que apoyan o desaprueban lo dicho. Enseguida, analizamos las expresiones de los tres estudiantes seleccionados, empleando para ello las categorías de análisis.

\subsubsection{Discurso de Gabriela}

El Cuadro 4 presenta el discurso de Gabriela organizado en tres expresiones.

\begin{tabular}{|l|l|}
\hline Expresión 1 & $\begin{array}{l}\text { No se intersecan y no tienen nada en su interior. [... }]^{3} \text { [No intersecarse significa] que sus líneas } \\
\text { no }(\ldots)^{4} \text { (hace una X con los dedos índices). }\end{array}$ \\
\hline Expresión 2 & $\begin{array}{l}\text { Profe, no se cortan así (cruza los brazos) sino así (mismo ademán de Nicolás en la Fotografía } \\
\text { 3). [...] Yo me acuerdo que [un polígono] no tiene (...) que no tiene líneas afuera. O sea, que } \\
\text { no, que no pasaba de ese punto. [No se intersecan] pues [es] que no sobresalen más líneas } \\
\text { después del punto común. }\end{array}$ \\
\hline Expresión 3 & $\begin{array}{l}\text { No, [los lados del polígono] sí se intersecan, pero no van más allá del punto que tienen en } \\
\text { común. }\end{array}$ \\
\hline
\end{tabular}

Cuadro 4 - Discurso de Gabriela

Fuente: elaborada por el autor

Rasgos para ser voz. Las expresiones de Gabriela son voz. Son inteligibles, aunque con varios sujetos tácitos, que se sobreentienden a la luz del intercambio en el que surgen y se confirman a partir de lo que parece estar comunicando sobre lo que significa que dos líneas se intersequen.

En el Cuadro 5 proponemos nuestras interpretaciones a cada expresión.

\begin{tabular}{|l|l|}
\hline Expresión 1 & $\begin{array}{l}\text { Afirma que los lados de un polígono (a los que denomina líneas) no se intersecan. Inferimos } \\
\text { que para ella la expresión se intersecan es aplicable al corte de dos rectas y no al de dos } \\
\text { segmentos. }\end{array}$ \\
\hline Expresión 2 & $\begin{array}{l}\text { Afirma que los segmentos } \overline{S T} \text { y } \overline{T R} \text {, (Figura 1) no se cortan como correspondería a una } \\
\text { intersección. Es decir, para ella hay al menos dos formas de cortarse y solo a una de ellas se } \\
\text { aplica la expresión se intersecan. Según ella, la intersección es una operación de cortarse, pero } \\
\text { no todo corte puede considerarse como una intersección. No basta con que haya un punto en } \\
\text { común; este no debe ser el extremo de los segmentos. Por eso niega que los segmentos se } \\
\text { intersequen, aunque se corten y el punto } T \text { sea común a los dos. Ella explicita su interpretación } \\
\text { acerca del tipo de objetos que intervienen en la operación de intersección: las rectas. }\end{array}$ \\
\hline
\end{tabular}

\footnotetext{
${ }^{3}$ Símbolo para indicar supresión de verbalización en la transcripción.

${ }^{4}$ Símbolo para indicar momento de silencio.
} 
Cambia de idea. Menciona que los segmentos sí se intersecan (reafirmando lo dicho por Nicolás, quien asegura que hay un punto de intersección). Sin embargo, ella aclara que el punto de corte está en el extremo de los segmentos. Esta aclaración es un indicio de la interpretación que empieza a reconstruir sobre la intersección, no solo como una acción recíproca entre rectas sino posible entre segmentos. Ahora intersecarse es sinónimo de cortarse $\mathrm{y}$, por tanto, tiene dos modos posibles. Los lados de un polígono no se intersecan así $\mathrm{X}$ sino así $\mathrm{V}$.

Cuadro 5 - Inteligibilidad de las expresiones de Gabriela

Fuente: elaborada por el autor

Además de inteligibles, las tres expresiones de Gabriela son auténticas. Los términos y gestos empleados, así como las construcciones gramaticales que hace son distintivos de la forma de hablar de una niña de su edad (Cuadro 6).

\begin{tabular}{|l|l|}
\hline Expresión 1 & $\begin{array}{l}\text { Al no encontrar cómo explicar qué significa para ella no intersecarse usa un gesto espontáneo } \\
\text { hecho con las manos (formando una X). }\end{array}$ \\
\hline Expresión 2 & $\begin{array}{l}\text { Se apoya, para explicar su idea, en una experiencia personal con la representación de un } \\
\text { polígono, y en gestos con las manos. }\end{array}$ \\
\hline Expresión 3 & Emplea frases idiosincráticas como "no van más allá". \\
\hline
\end{tabular}

Cuadro 6 - Autenticidad de las expresiones de Gabriela Fuente: elaborada por el autor

Rasgos de la voz. En el Cuadro 7 sintetizamos rasgos de la voz de Gabriela. Su narrativa es dubitativa sobre lo que significa que los lados de un polígono se intersequen.

\begin{tabular}{|c|c|}
\hline $\begin{array}{l}\text { Especialización } \\
\text { del vocabulario }\end{array}$ & $\begin{array}{l}\text { Combina términos coloquiales y matemáticos, apoyándose en representaciones con las } \\
\text { manos. Por ejemplo, dice: "intersecan", "polígono" y "punto en común", junto con } \\
\text { expresiones como "no tiene nada en su interior", "no tiene líneas afuera" o "no sobresalen } \\
\text { más líneas". Usa línea en lugar de lado. } \\
\text { Introduce en la conversación el término intersecan, en plural, con lo que entrevemos que } \\
\text { ella conoce, de antemano, que se trata de una operación entre objetos. Pero antes de escuchar } \\
\text { a sus compañeros, en lugar de referirse a la existencia de un punto en común, como criterio } \\
\text { de verificación de la operación de intersección entre objetos, ella usa el término se cortan } \\
\text { para explicar cuándo dos líneas se intersecan, acompañado de la representación con los } \\
\text { dedos en cruz. En ese sentido, es posible que inicialmente tenga asociada la palabra } \\
\text { intersecantes a un cierto tipo de objetos, las rectas. }\end{array}$ \\
\hline Objetificación & $\begin{array}{l}\text { Aún no concibe la intersección como una operación bien definida entre dos objetos } \\
\text { geométricos cualesquiera, sino, de forma aún muy limitada, como un tipo de configuración. } \\
\text { En su aprendizaje ella debería ampliar el conjunto de objetos que se pueden operar mediante } \\
\text { la intersección, así como pasar de considerar la intersección como acción física } \\
\text { bidireccional entre objetos particulares a verla como un conjunto definido en términos de } \\
\text { pertenencia a los dos objetos que se operan, para en un futuro, nominalizar la operación de } \\
\text { intersección, y predicar sobre ella. } \\
\text { Además, en el proceso de transición discursiva, debería incluir atributos de cantidad para } \\
\text { especificar de forma explícita a cuántos segmentos se está refiriendo. El empleo de frases } \\
\text { como: "sus líneas no (...) (hace una X con los dedos índices) se [cruzan]" dificultan la } \\
\text { comunicación de la idea que quiere expresar. }\end{array}$ \\
\hline $\begin{array}{l}\text { Mediadores } \\
\text { visuales }\end{array}$ & $\begin{array}{l}\text { La segunda expresión incorpora un gesto en el discurso para remplazar la frase se cruzan. } \\
\text { Este gesto es parte de su expresión. En cambio, los otros gestos que acompañan sus } \\
\text { expresiones orales (cruzar los dedos y unir las yemas de los dedos) son un apoyo para } \\
\text { comunicarse, aunque menos sofisticados que la representación del profesor, en la que } \\
\text { también ella se apoya. }\end{array}$ \\
\hline $\begin{array}{l}\text { Acciones } \\
\text { rutinarias }\end{array}$ & $\begin{array}{l}\text { Está aprendiendo a usar el criterio para decidir cuándo afirmar que dos objetos se intersecan. } \\
\text { Este criterio aún no es una rutina que ella haya incorporado como un patrón de actividad } \\
\text { matemática. En las expresiones se ve un avance al ir considerando la existencia de un punto } \\
\text { en común en la decisión. Pero en la Expresión } 3 \text { se aprecia cierto recelo en emplear la frase, } \\
\text { puesto que la representación de los segmentos } \overline{S T} \text { y } \overline{T R} \text { no se ajusta a las representaciones } \\
\text { que ella recuerda, de objetos que se intersecan. Su narrativa: "No, sí se intersecan, pero no }\end{array}$ \\
\hline
\end{tabular}


van más allá del punto que tienen en común" - es un indicio de ello.

Cuadro 7 - Rasgos de la voz de Gabriela

Fuente: elaborada por el autor

Efecto de la voz de Gabriela en la construcción colectiva o compartida de significado.

En el Cuadro 8 describimos el efecto de la voz de Gabriela.

\begin{tabular}{|c|c|}
\hline $\begin{array}{l}\text { Inteligibilidad } \\
\text { para } \\
\text { participantes en } \\
\text { el discurso }\end{array}$ & $\begin{array}{l}\text { La voz es inteligible para otros compañeros de la clase. En particular, Tomás y Nicolás } \\
\text { siguen de cerca lo que dice y reaccionan a sus expresiones. Nicolás expresa con gestos de } \\
\text { cara y manos lo que ella dice. Tomás expone su interpretación e incluso, en un momento } \\
\text { dado, manifiesta no haberla entendido, por lo cual Gabriela explica su idea. }\end{array}$ \\
\hline Voz compartida & $\begin{array}{l}\text { Es una voz compartida, aunque no por todos los estudiantes de la clase. Con posterioridad } \\
\text { al intercambio relatado, el profesor vuelve a preguntar si los segmentos }(\overline{S T} \text { y } \overline{T R}) \text { se } \\
\text { intersecan, y no hay unidad en las respuestas. Pero, su voz sí es compartida por Tomás, } \\
\text { Nicolás y otros niños que no tienen un lugar protagónico en el intercambio pero que } \\
\text { aprueban lo que ella dice. Su voz representa la de estos niños, aunque ella, en lugar de usar } \\
\text { la expresión se cruzan, previamente empleada por Tomás, hace un parafraseo usando se } \\
\text { cortan, frase que tiene un significado más cercano a la idea de detenerse o colisionar que } \\
\text { de interferir. En el caso de los segmentos } \overline{S T} \text { y } \overline{T R} \text {, el punto T está en un extremo, por lo } \\
\text { que detiene al continuo de puntos que conforman el segmento, pues este llega hasta ahí. } \\
\text { El despliegue de su voz nos permite afirmar que ella genera oportunidades para que sus } \\
\text { compañeros expresen sus interpretaciones de la relación intersecarse y a la vez ella se vale } \\
\text { de lo que dicen ellos para cambiar de idea de lo que significa. }\end{array}$ \\
\hline Voz colectiva & $\begin{array}{l}\text { Ella pone el tema de conversación, secundada por el profesor. Pero su voz es colectiva por } \\
\text { cuanto la estudiante va incorporando en sus expresiones ideas sugeridas por Nicolás y } \\
\text { Tomás, lo que incluso la lleva a cambiar su interpretación inicial acerca de la intersección } \\
\text { entre los segmentos } \overline{S T} \text { y } \overline{T R} \text { o entre dos lados de un polígono. Ella expresa sus ideas al } \\
\text { respecto y se muestra atenta a lo que dicen sus compañeros. Esto la lleva a considerar la } \\
\text { existencia de un punto en común, sugerida por Tomás y representada por Nicolás, } \\
\text { identificando un nuevo punto de vista sobre la situación, inadvertido antes. Al explicar su } \\
\text { nueva perspectiva, admite la posibilidad de que los segmentos se intersequen, aunque con } \\
\text { reservas. }\end{array}$ \\
\hline
\end{tabular}

Cuadro 8 - Efecto de la voz de Gabriela

Fuente: elaborado por el autor

\subsubsection{Discurso de Tomás}

Tomás formula cuatro oraciones, apoyado en gestos de mano y boca, a lo largo de la interlocución. Las reunimos en dos expresiones, según la intención comunicativa (Cuadro 9).

\begin{tabular}{|l|l|}
\hline Expresión 1 & No tiene un punto en común para (gesto con los dedos formando una cruz). \\
\hline Expresión 2 & $\begin{array}{l}\text { [Intersecarse es] que al cruzarse queda un punto ahí (señala con la boca el lugar donde quedaría } \\
\text { la intersección de los dedos; Figura 2). Cuando se cruzan el punto es ese. }\end{array}$ \\
\hline
\end{tabular}

Cuadro 9 - Discurso de Tomás

Fuente: elaborada por el autor

Rasgos para ser voz. Las expresiones de Tomás son voz matemática. Son inteligibles, aunque los sujetos son tácitos, y auténticas. En el Cuadro 10 presentamos nuestras interpretaciones.

Expresión 1 $\quad$ Afirma que los segmentos $\overline{S T}$ y $\overline{T R}$ no se intersecan pues él no ve un punto en común que permita afirmar que los segmentos se crucen; es decir, para él, el punto $\mathrm{T}$ no determina dos segmentos o un segmento y una semirrecta en ninguno de los segmentos originales. 
Expresión 2 Sostiene que la intersección refiere a un punto en común que debe cumplir una condición relativa a su posición en cada uno de los segmentos, a saber, debe quedar en el interior de cada uno de ellos y no en el extremo.

Cuadro 10 - Inteligibilidad de las expresiones de Tomás

Fuente: elaborada por el autor

La voz de Tomás es auténtica. Introduce de manera espontánea la expresión punto en común, que es empleada luego por varios compañeros (Cuadro 11).

\begin{tabular}{|l|l|}
\hline Expresión 1 & $\begin{array}{l}\text { Introduce la expresión punto en común, que es usada luego por Gabriela y otros estudiantes, y } \\
\text { alude a que los segmentos } \overline{S T} \text { y } \overline{T R} \text { no se cruzan, haciendo un gesto con las manos. }\end{array}$ \\
\hline Expresión 2 & Usa expresiones del lenguaje común como "queda un punto ahí" o "cuando se cruzan". \\
\hline
\end{tabular}

Cuadro 11 - Autenticidad de las expresiones de Tomás

Fuente: elaborada por el autor

Rasgos de la voz. En el Cuadro 12 presentamos lo rasgos de la voz de Tomás. En su narrativa emite expresiones categóricas para argumentar por qué los segmentos $\overline{S T}$ y $\overline{T R}$ no se intersecan.

\begin{tabular}{|l|l|}
\hline $\begin{array}{l}\text { Especialización } \\
\text { del vocabulario }\end{array}$ & $\begin{array}{l}\text { Combina términos coloquiales y matemáticos, apoyándose en representaciones con las } \\
\text { manos. Emplea la expresión punto en común y la relaciona con la intersección. Pero, no lo } \\
\text { hace como criterio para justificar que los segmentos se intersecan, sino para afirmar que no } \\
\text { lo hacen. Según él, el punto en común debería producirse en el interior de los segmentos, } \\
\text { como efecto del cruce entre ellos. }\end{array}$ \\
\hline Objetificación & $\begin{array}{l}\text { El proceso de objetificación es incipiente. Confiere a los segmentos poder de acción. Al } \\
\text { igual que en el caso de Gabriela, Tomás debería transitar hacia el reconocimiento de una } \\
\text { operación entre objetos geométricos, no necesariamente rectas y, en un futuro hacia una } \\
\text { relación entre objetos matemáticos de diferente índole. }\end{array}$ \\
\hline $\begin{array}{l}\text { Mediadores } \\
\text { visuales }\end{array}$ & $\begin{array}{l}\text { Usa, principalmente, como mediadores visuales, gestos de brazos, dedos de las manos y } \\
\text { boca. Con brazos y dedos corporeiza los segmentos y con la boca señala lo que sería el } \\
\text { punto de intersección, si los segmentos se cruzaran. }\end{array}$ \\
\hline $\begin{array}{l}\text { Acciones } \\
\text { rutinarias }\end{array}$ & $\begin{array}{l}\text { La búsqueda de un punto en común aún no se constituye en una rutina apropiada para } \\
\text { verificar si dos segmentos se intersecan pues el estudiante agrega condiciones a la } \\
\text { comunalidad del punto. Además de pertenecer a ambas líneas, el punto debería pertenecer } \\
\text { al interior de los segmentos. }\end{array}$ \\
\hline
\end{tabular}

Cuadro 12 - Rasgos de la voz de Tomás

Fuente: elaborada por el autor

Efecto de la voz de Tomás en la construcción colectiva o compartida de significado. En

el Cuadro 13 presentamos el efecto de la voz de Tomás.

\begin{tabular}{|l|l|}
\hline $\begin{array}{l}\text { Inteligibilidad } \\
\text { para } \\
\text { participantes en } \\
\text { el discurso }\end{array}$ & $\begin{array}{l}\text { La voz es inteligible para otros compañeros de la clase. Es usada por Gabriela en sus } \\
\text { explicaciones posteriores a la primera verbalización del estudiante, refiriéndose a un punto } \\
\text { en común. Y gracias a ella Nicolás acepta (como veremos) que sí hay un punto en común } \\
\text { entre los segmentos } \overline{S T} \text { y } \overline{T R} \text {. Otros estudiantes consultados por el profesor, cuya } \\
\text { participación discursiva es menor, repiten que los segmentos no se intersecan porque no } \\
\text { hay un punto en común. }\end{array}$ \\
\hline Voz compartida & $\begin{array}{l}\text { Es una voz compartida pues expresa una interpretación representativa de lo que piensan } \\
\text { varios estudiantes. Además, porque en la transcripción del trabajo que hicieron los } \\
\text { estudiantes cuando examinaban ejemplos y no ejemplos de polígonos, más de uno empleó } \\
\text { la expresión punto en común para referirse a atributos de los casos que observaban. }\end{array}$ \\
\hline Voz colectiva & $\begin{array}{l}\text { La voz es colectiva. Incluye el término cruzarse propuesto por un compañero y el gesto } \\
\text { de Gabriela. }\end{array}$ \\
\hline
\end{tabular}

Cuadro 13 - Efecto de la voz de Tomás

Fuente: elaborado por el autor 


\subsubsection{Discurso de Nicolás}

Nicolás, expresándose principalmente con gestos de cara y manos, interviene en la conversación e influye en el cambio de interpretación que experimenta Gabriela y, en general, varios estudiantes. Sintetizamos las intervenciones de Nicolás en el Cuadro 14.

\begin{tabular}{|l|l|}
\hline Expresión 1 & $\begin{array}{l}\text { Asiente con la cabeza cuando Gabriela menciona que los lados de los polígonos no se } \\
\text { intersecan.) [...] (Hace un gesto con las manos, cuando Cristian y Tomás mencionan que } \\
\text { los segmentos no se intersecan.) (Figura 3). }\end{array}$ \\
\hline Expresión 2 & (Hace un gesto de manos (Figura 4) mientras habla.) ¡Ah, pues sí! \\
\hline Expresión 3 & Sí, porque se intersecan en $T$. \\
\hline
\end{tabular}

Cuadro 14 - Discurso de Nicolás

Fuente: elaborada por el autor

Rasgos para ser voz. Las expresiones de Nicolás, surgidas en interacción comunicativa con sus compañeros, son voz, aunque su narrativa sea principalmente gestual. En el Cuadro 15 presentamos nuestras interpretaciones que nos permiten afirmar que sus expresiones son inteligibles.

\begin{tabular}{|l|l|}
\hline Expresión 1 & $\begin{array}{l}\text { Inicialmente coincide con Gabriela al considerar que los segmentos }(\overline{S T} \text { y } \overline{T R} \text { ) no se intersecan } \\
\text { porque no se cortan como lo hacen las rectas intersecantes, es decir, no se cruzan. }\end{array}$ \\
\hline Expresión 2 & $\begin{array}{l}\text { Cuando Tomás menciona la expresión “puntos en común” (que es repetida por el profesor), } \\
\text { cambia de idea. La cámara parece haber captado el momento en el que cae en cuenta que el } \\
\text { punto T es común a los dos segmentos y no requiere de la condición de ser interior a estos. }\end{array}$ \\
\hline Expresión 3 & $\begin{array}{l}\text { Cuando el profesor pregunta: “¿Entonces habrá intersección o no habrá intersección?” cristaliza } \\
\text { la idea diciendo: "Sí, porque se intersecan en T”. }\end{array}$ \\
\hline
\end{tabular}

Cuadro 15 - Inteligibilidad de las expresiones de Nicolás

Fuente: elaborada por el autor

Las expresiones de Nicolás, principalmente gestuales, son auténticas. En el Cuadro 16 las describimos.

\begin{tabular}{|c|l|}
\hline Expresión 1 & El gesto que hace con las manos (Figura 4) es propio del estudiante, es el primero que lo hace. \\
\hline Expresión 2 & Además del gesto que hace con las manos expresa sorpresa de forma evidente. \\
\hline Expresión 3 & $\begin{array}{l}\text { Argumenta, por voluntad propia, que los segmentos son intersecantes diciendo en qué punto se } \\
\text { intersecan. }\end{array}$ \\
\hline
\end{tabular}

Cuadro 16 - Autenticidad de las expresiones de Nicolás

Fuente: elaborada por el autor

Rasgos de la voz. En el Cuadro 17 presentamos los rasgos de la voz de Nicolás. Destacamos la Expresión 3, pues es una narrativa sustentada o respaldada por la precisión de cuál es el punto de intersección, en una frase especializada en la que usa apropiadamente el término se intersecan. Es una descripción del resultado del proceso de intersecar.

\begin{tabular}{|l|l|}
\hline $\begin{array}{l}\text { Especialización } \\
\text { del vocabulario }\end{array}$ & $\begin{array}{l}\text { Emplea vocabulario especializado para justificar por qué los segmentos } \overline{S T} \text { y } \overline{T R} \text { son } \\
\text { intersecantes. }\end{array}$ \\
\hline Objetificación & $\begin{array}{l}\text { La narrativa de Nicolás en la Expresión } 3 \text { refleja un grado de objetificación mayor que el } \\
\text { de Gabriela y Tomás, al hacer mención a un objeto matemático producto de la intersección. }\end{array}$ \\
\hline $\begin{array}{l}\text { Mediadores } \\
\text { visuales }\end{array}$ & $\begin{array}{l}\text { Se apoya en las expresiones gestuales de Gabriela y Tomás, además de la representación de } \\
\text { los segmentos que hace el profesor. }\end{array}$ \\
\hline
\end{tabular}


Cuadro 17 - Rasgos de la voz de Nicolás

Fuente: elaborada por el autor

Efecto de la voz de Nicolás en la construcción colectiva o compartida de significado.

En el Cuadro 18 nos referimos al efecto de la voz de Nicolás.

\begin{tabular}{|l|l|}
\hline $\begin{array}{l}\text { Inteligibilidad para } \\
\text { participantes en el } \\
\text { discurso }\end{array}$ & $\begin{array}{l}\text { La voz es inteligible para otros compañeros de la clase. El gesto que usa es empleado } \\
\text { por otros compañeros. Su argumentación es escuchada y usada por Gabriela y Tomás. }\end{array}$ \\
\hline Voz compartida & Es una voz compartida. Provoca una transición discursiva sobre la intersección. \\
\hline Voz colectiva & $\begin{array}{l}\text { La voz es colectiva. A partir de lo que dicen Gabriela y Tomás, él y otros compañeros } \\
\text { admiten que la operación de intersección puede darse también entre segmentos y que } \\
\text { el punto de intersección no requiere ninguna condición con respecto a la posición en } \\
\text { los objetos que se intersecan. }\end{array}$ \\
\hline
\end{tabular}

Cuadro 18 - Efecto de la voz de Nicolás

Fuente: elaborado por el autor

\section{Discusión}

El análisis realizado a un conjunto de expresiones inteligibles en el intercambio comunicativo escogido nos permitió profundizar en la comunicación que se dio en un breve lapso de la clase de geometría observada. Corroboramos resultados investigativos que afirman que los estudiantes tienen opción de escuchar, decir términos matemáticos, expresarse (BARWELL, 2013; CLARKE; XU; WAN, 2013) y compartir sus interpretaciones (JABLONKA, 2006; CAO et al., 2013; KAUR, 2013; INGRAM; ANDREWS; PITT, 2019).

Sin pretender afirmar que los tres estudiantes (en los que centramos el análisis) lograron una producción discursiva totalmente clara, coherente y completa, sí apreciamos un espacio de participación discursiva, generado por el profesor, donde pudieron expresar sus significados y los contrastaron con los de sus compañeros, constituyéndose en voces. No fue un simple intercambio de palabras, fue una participación discursiva real. El vocabulario, los giros lingüísticos, los gestos que acompañan lo que dicen los estudiantes Gabriela, Tomás y Nicolás, y el esfuerzo evidente por involucrarse de manera pertinente en la conversación promovida por el profesor, todo ello refleja autenticidad (Cuadros 6, 11 y 16). Además, encontramos evidencias de construcción colectiva y de generación de significados compartidos sobre la intersección (Cuadros 8, 13 y 18).

Adicionalmente, al optar por hacer un análisis interpretativo de las expresiones discursivas, aprovechando el modelo propuesto por Sfard (2008) para estudiar los rasgos de la voz, nuestro estudio aporta una mirada en detalle a la construcción de significado de la 
operación intersección, haciendo un seguimiento detallado a una pequeña transición discursiva, ejercicio que no hemos visto reportado en estudios previos que se enfocan en interacciones comunicativas en el aula de clase. En ese sentido, ilustramos la potencia del modelo para hacer seguimiento al aprendizaje.

Aunque los significados exhibidos por los estudiantes distan del significado matemático de intersección, evolucionan, en la dinámica discursiva, en tres sentidos: hacia considerar la intersección como una operación matemática, más que como un atributo relativo a la posición de dos rectas o una acción física protagonizada por objetos geométricos; hacia ampliar el dominio de objetos a los que es aplicable la operación de intersecarse; y hacia generar como rutina, el criterio de verificación o regla de construcción de la determinación de al menos un punto de intersección, más allá de percibir o buscar una configuración en X.

Lo anterior nos lleva a afirmar que los estudiantes aprendieron ideas matemáticas sobre la intersección, gracias a la posibilidad de participar en el discurso del aula, desplegando su voz. Como señalan Morgan y Sfard (2016) la participación discursiva contribuyó a que los estudiantes avanzaran en su aprendizaje transitando desde expresiones del uso común del lenguaje como cruzarse, cortarse, líneas que sobresalen y una representación prototípica de dos líneas intersecantes, hacia expresiones como punto en común y el punto de intersección es $T$ y una ampliación de la representación en la que tienen cabida segmentos con un extremo en común, como intersecantes.

El uso del lenguaje común como punto de partida fue importante para promover los cambios que se observaron en las voces de los estudiantes. Infortunadamente, el hecho de que los estudiantes no tengan un procedimiento visual especializado de reconocimiento de objetos intersecantes hace que el proceso de objetificación de la operación intersección de objetos geométricos no haya avanzado considerablemente.

Afirmamos que los resultados del estudio apoyan los llamados a explicitar qué significados tienen los estudiantes de términos matemáticos y contrastarlos con el uso canónico (MORGAN; SFARD, 2016). Por ejemplo, si no se hubiera ahondado en la expresión se intersecan, proferida por Gabriela, no se podría haber identificado lo que ella quería decir. El estudio muestra que al menos tres niños que tuvieron voz en la clase tenían significados distintos a los "oficiales", situación que apoya la recomendación de detenerse en la clase para ahondar en los significados y lograr una comunicación más productiva.

Probablemente, el tiempo destinado a tales actividades lentificó el trabajo previsto sobre polígonos e implicó dedicar más sesiones de clase que las planeadas. Pero al escuchar a los estudiantes y permitirles el despliegue de su voz, el profesor generó un espacio útil de 
participación discursiva que ilustra el potencial de la interacción comunicativa en la construcción de significado y que consideramos estimulante para que otros profesores se animen a propiciarlo.

\section{Conclusiones}

La herramienta analítica construida a partir de nuestra conceptualización de voz y el modelo de Sfard (2008) para los componentes del discurso nos permitió ahondar en las expresiones de cada estudiante. Además de tener criterios para afirmar cuándo una expresión es voz, pudimos identificar rasgos de la expresión en los que vemos indicios del proceso de transición discursiva de personas que están aprendiendo qué significa intersecarse, e identificar cuáles voces son colectivas y cuáles son compartidas. En particular, el vocabulario y la sintaxis que manejan los estudiantes nos permitieron ahondar en el proceso de objetificación de la intersección y estudiar su complejidad.

Urge construir una base de conocimiento profesional para quienes asuman el desafío que implica hacer de las aulas espacios de participación discursiva donde las voces de los estudiantes tengan un papel protagónico en la construcción de significado. Estudios como el nuestro aportan luces sobre cómo hacer realidad esta propuesta en las aulas. Particularmente, en la preparación de tareas y de la gestión en el aula, los profesores pueden valerse de estudios como este para anticipar posibles intervenciones de estudiantes y prever cómo darles oportunidades de construir significados colectivos y compartidos.

No nos detuvimos en la gestión del profesor pues nuestro interés era rastrear y caracterizar voces de estudiantes. Pero es evidente que estas surgieron gracias al espacio propiciado por el profesor, que permitió la interacción discursiva sobre la intersección, a pesar de no ser el tema previsto para la clase. Nos preguntamos qué otras acciones del profesor propiciaron en este episodio el surgimiento de las voces. Creemos que es un asunto que debe estudiarse detenidamente; sería interesante complementar el estudio haciendo un análisis en profundidad de la gestión del profesor enfocada en la gestión de las voces.

\section{Referencias}

BARWELL, R. The academic and the everyday in mathematicians' talk: The case of the hyperbagel. Language and Education, Londres, v. 27, n. 3, 2012, p. 207-222.

BEN-ZVI, D.; SFARD, A. Ariadna's thread, Daedalus' wings, and the learner's autonomy. Éducation et Didactique, Bretagne, v. 1, n. 3, 2007, p. 123-141. 
CAMARGO, L. Estrategias cualitativas de investigación en Educación Matemática. Bogotá: Fondo de Publicaciones Universidad Pedagógica Nacional, en evaluación.

CAO, Y.; GUO, K.; DING, L.; MOK, I. A. C. Students at the front: Examples from a Beijing classroom. En: KAUR, B.; ANTHONY, G.; OHTANI, M; CLARKE, D. (ed.). Student voice in mathematics classrooms around the world. Roterdam: Sense Publishers, 2013. p. 33-52.

CLARKE, D.; XU, L.H.; WAN, M.E.V. Spoken mathematics as an instructional strategy: The public discourse of mathematics classrooms in different countries. En: KAUR, B.; ANTHONY, G.; OHTANI, M; CLARKE, D. (ed.). Student voice in mathematics classrooms around the world. Roterdam: Sense Publishers, 2013. p. 13-32.

ESMONDE, I; LANGER-OSUNA, J. Power in numbers: Student participation in mathematical discussions in heterogeneous spaces. Journal for Research in Mathematics Education, Reston, v. 44, n.1, 2013, p. 288-315.

INGRAM, J. Moving forward with ethnomethodological approaches to analysing mathematics classroom interactions. ZDM Mathematics Education, Hamburgo, v. 50, 2018, p. 1065-1075.

INGRAM, J.; ANDREWS, N.; PITT. A. When students offer explanations without the teacher explicitly asking them to. Educational Studies in Mathematics, Utrecht, v. 101, 2019, p. 51-66.

JABLONKA, E. Student(s) at the front: Forms and functions in six classrooms from Germany, Hong Kong and the United States. En: CLARKE, D. J.; EMANUELSSON, J.; JABLONKA, E.; MOK, I. A. C. (ed.). Making connections: Comparing mathematics classrooms around the world. Rotterdam: Sense Publishers, 2006. p. 107-126.

JUNG J.; SHÜTTE, M. An interactionist perspective on mathematics learning: Conditions of learning opportunities in mixed-ability groups within linguistic negotiation processes. ZDM Mathematics Education, Hamburgo, v. 50, 2018, p. 1089-1099.

KAUR, B. Participation of students in content-learning classroom discourse: A study of two grade 8 mathematics classes in Singapore. En: KAUR, B.; ANTHONY, G.; OHTANI, M.; CLARKE, D. (ed.). Student voice in mathematics classrooms around the world. Rotterdam: Sense Publishers, 2013. p. 65-88.

MORGAN, C.; SFARD, A. Investigating changes in high-stakes mathematics examinations: A discursive approach. Research in Mathematics Education. London, v. 18, n. 2, 2016, p. 92-119.

RADFORD, L.; BARWELL, R. Language in mathematics education research. En: GUTIÉRREZ, Á., LEDER, G.C., BOERO, P. (ed.). The second handbook of research on the psychology of mathematics education: The journey continues. Rotterdam: Sense Publishers, 2016. p. 275-313.

SFARD, A. Sobre las metáforas de la adquisición y de la participación para el aprendizaje de las matemáticas. En: SFARD, A. (ed.). Aprendizaje de las matemáticas escolares desde un enfoque comunicacional. Cali: Universidad del Valle, 2008. p. 23-37.

VILLALTA, M.A. Análisis de la conversación. Una propuesta para el estudio de la interacción didáctica en sala de clase. Estudios Pedagógicos, Valdivia, v. 35, n.1, 2009, p. 221-238.

WALSHAW, M.; ANTHONY, G. The teacher's role in classroom discourse: A review of recent research into mathematics classrooms. Review of Educational Research, Pensilvania, v. 78, n. 3, 2008, p. 516-551.

WOOD, M.; KALINEC, C. A. Student talk and opportunities for mathematical learning in small group 
interactions. International Journal of Educational Research, Belfast, v. 51 - 52, 2012, p. 109-127.

Submetido em 02 de Dezembro de 2019. Aprovado em 02 de Julho de 2020. 